\title{
A Five-Port Deembedding Method for Floating Two-Port Networks
}

\author{
Reza Mahmoudi and Joseph L. Tauritz, Member, IEEE
}

\begin{abstract}
Radio frequency (RF) high-power bipolar transistors are often constructed with the collector accessible at the bottom of the device. Characterization is carried out on substrate mounted devices. The classical methods developed for the "on wafer" capacitance-voltage $(\mathrm{CV})$ and alternating current (ac) measurement of grounded devices are then no longer applicable. A (general) deembedding algorithm is presented in which the medium surrounding the transistor is taken to be a generic five port and the transistor is treated as a floating two port. Using this approach, one can model a wide variety of configurations, including coupled lines, bondwire complexes with mutual coupling, vias and packages enabling one pass deembedding. Use of this algorithm facilitates an integrated approach improving accuracy and speed. Implementation of the five-port algorithm in HP's-microwave design software, MDS, and HP's parameter extraction software, IC-CAP as well as its application to highfrequency power transistor modeling are described.
\end{abstract}

Index Terms-Admittance matrix, five-port circuits, microwave amplifiers, microwave measurement.

\section{INTRODUCTION}

A CCURATE extraction of parameter sets representative of an active device's electrical performance is an activity of increasing importance to high-frequency specialists.

Usually the collector of a radio frequency (RF) high-power transistor is accessible through the bottom of the device so that the transistor must be mounted on a substrate, often a PC board, for characterization (see Fig. 1). In practice, an assortment of lumped and distributed components may be present between the reference planes at which the measurement is carried out and the active device (the transistor).

Standard deembedding procedures (see [1] and [2]) require the use of quite a number of matrix manipulations (involving $Y, Z$ and $A B C D$ parameters). In many cases, the very complexity of the environment makes simplification of the equivalent circuit mandatory decreasing implicitly the accuracy of the deembedded data. In order to avoid the use of unhandy procedures and to maintain the integrity of the equivalent circuit a new solution to the general deembedding problem has been sought.

In this work, the transistor mounting environment is treated as a generic five port and the problem of deembedding the

Manuscript received July 20, 1997; revised December 4, 1998.

The authors are with the Microwave Component Group, Delft University of Technology (DIMES/ECTM), Delft 2600 GB The Netherlands (e-mail: rmahmoudi@et.tudelft.nl).

Publisher Item Identifier S 0018-9456(98)09847-7.

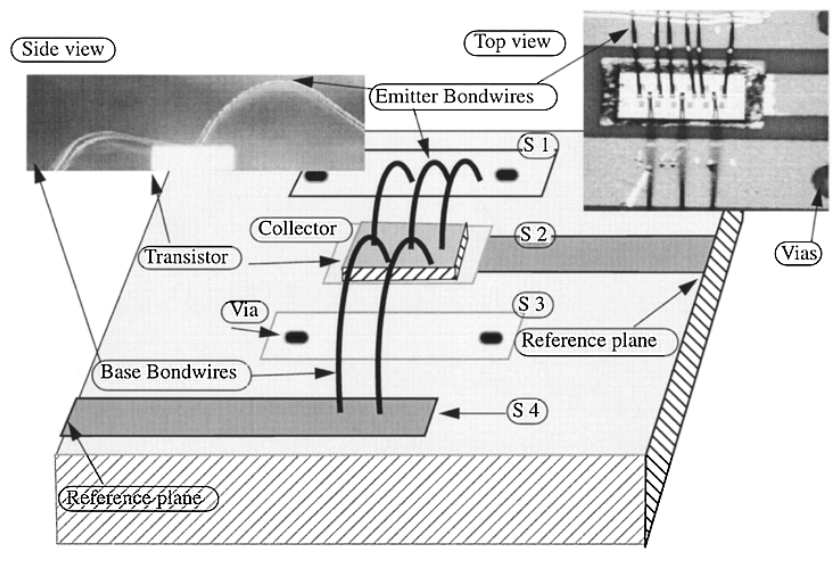

Fig. 1. A standard layout for the mounting and measurement of power transistors.

measured data back to the internal terminals of the active devices considered in some depth.

\section{A General Expression for Five-Port DeEmbedDing}

In order to derive a general expression for deembedding, we take in Fig. 2.

- The active device mounting environment is a five port, represented by the mathematically symmetrical impedance matrix $Z_{\text {Five }}$. Two ports of the five port are external ports and the other three are treated as internal ports.

- The transistor is a "floating two-port network" represented by $Z_{\text {Tran }}$.

- The mounted transistor is a grounded two port represented by $Z_{\text {Meaured }}$.

The above $n$-ports can be defined as

$$
\begin{aligned}
v_{\text {Five }, i} & =\sum_{j=1}^{5} Z_{\text {Five },[i, j]} \bullet i_{\text {Five }, j} \\
v_{\text {Tran }, i} & =\sum_{j=1}^{2} Z_{\operatorname{Tran}[i, j]} \bullet i_{\operatorname{Tran}, j} \\
v_{\text {Measured }, i} & =\sum_{j=1}^{2} Z_{\text {Measured }[i, j]} \bullet i_{\text {Measured }, j} .
\end{aligned}
$$

Our aim is to derive an expression for $Z_{\text {Tran }}$ as a function of $Z_{\text {Five }}$ and $Z_{\text {measured. }}$. In Fig. 2 we identify nine boundary 


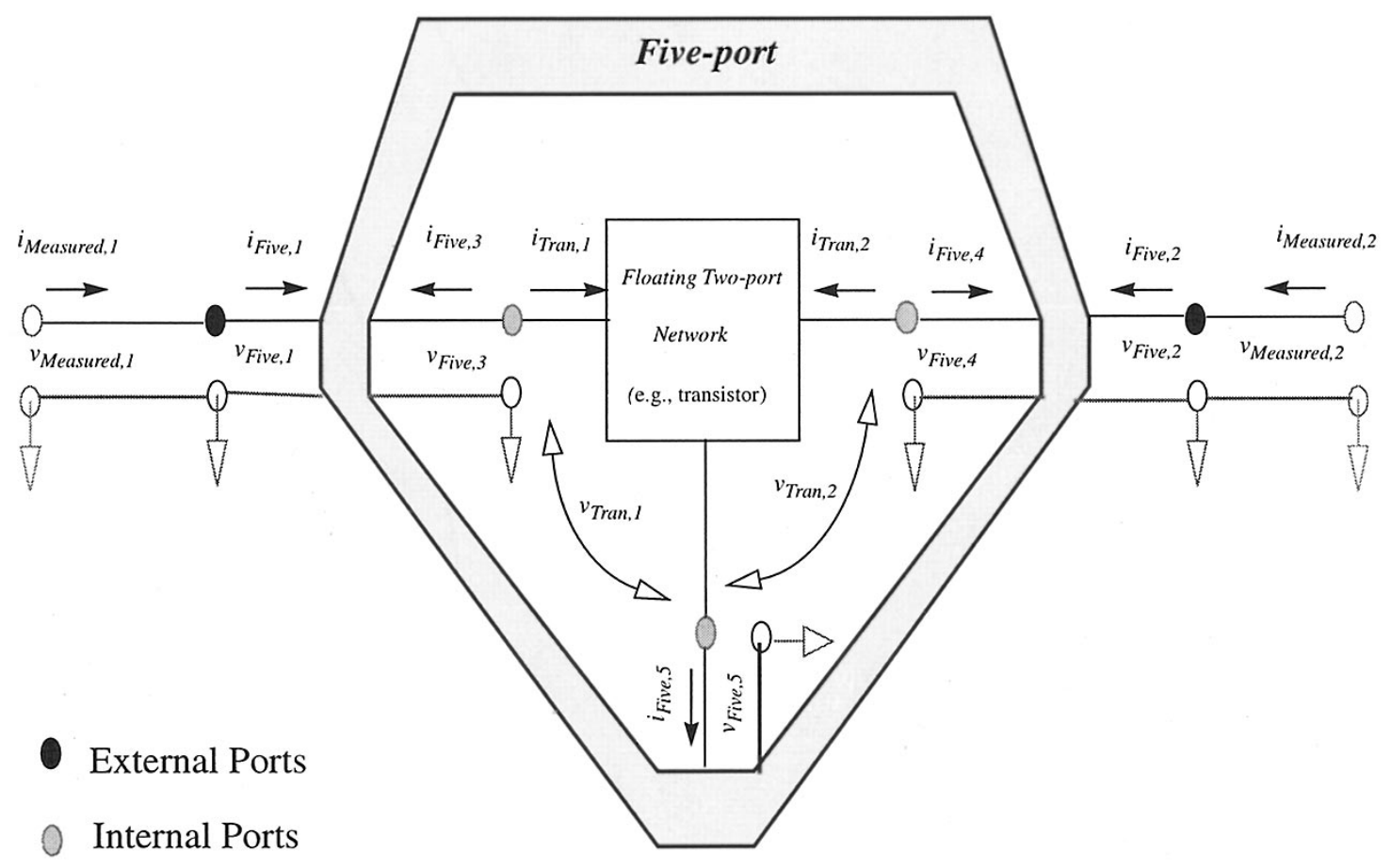

Fig. 2. Illustration of a transistor embedding in the five port.

conditions, namely

$$
\begin{aligned}
& i_{\text {Five }, 1}=i_{\text {Measured }, 1} \\
& i_{\text {Five }, 2}=i_{\text {Measured }, 2} \\
& i_{\text {Five }, 3}=-i_{\text {Tran }, 1} \\
& i_{\text {Five }, 4}=-i_{\text {Tran }, 2} \\
& i_{\text {Five }, 5}=-i_{\text {Five }, 4}-i_{\text {Five }, 3} \\
& v_{\text {Tran }, 1}=v_{\text {Five }, 3}-v_{\text {Five }, 5} \\
& v_{\text {Tran }, 2}=v_{\text {Five }, 4}-v_{\text {Five }, 5} \\
& v_{\text {Five }, 1}=v_{\text {Measured }, 1} \\
& v_{\text {Five }, 2}=v_{\text {Measured }, 2} .
\end{aligned}
$$

These relations lead to a set of nonlinear and frequency independent equations "F" (1) derived using the symbolic computation program MAPLE, in which the impedance matrix of the transistor is expressed in terms of the impedance matrix of the environment $Z_{\text {Five }}$, as well as the impedance matrix of the measured data $Z_{\text {Measured }}$ as shown in (1) at the bottom of the page (see Appendix).

\section{The Deembedding Procedure}

The procedure which is used to carry out the deembedding is outlined in Fig. 3. To initialize the deembedding procedure two preparatory steps are required.

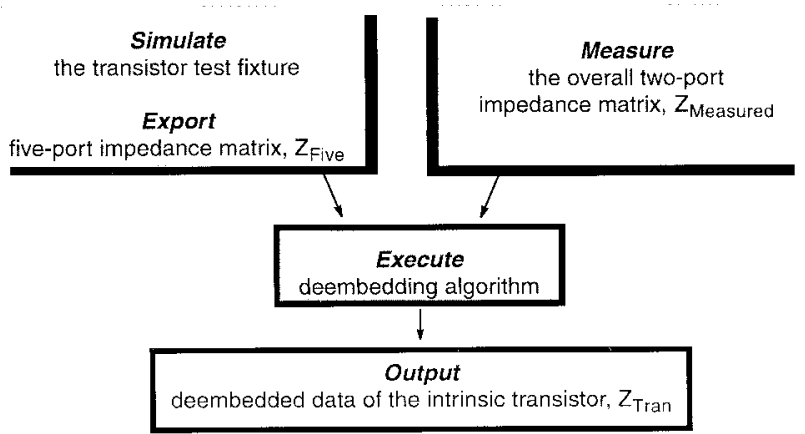

Fig. 3. The deembedding prodecure.

1) The impedance matrix of the five-port dataset which is representative of the three terminal network's mounting environment is generated as a function of frequency and written to a file.

2) The overall two-port impedance matrix of the mounted three terminal network is measured at the same frequencies as in the previous step.

Using this approach, one is able to deembed the measured data. The accuracy of the processed data is dependent on

- the appropriateness of the assumed equivalent circuit,

- the accuracy of the models used to represent the elements of the equivalent circuit, and

- the accuracy of the simulation program. 


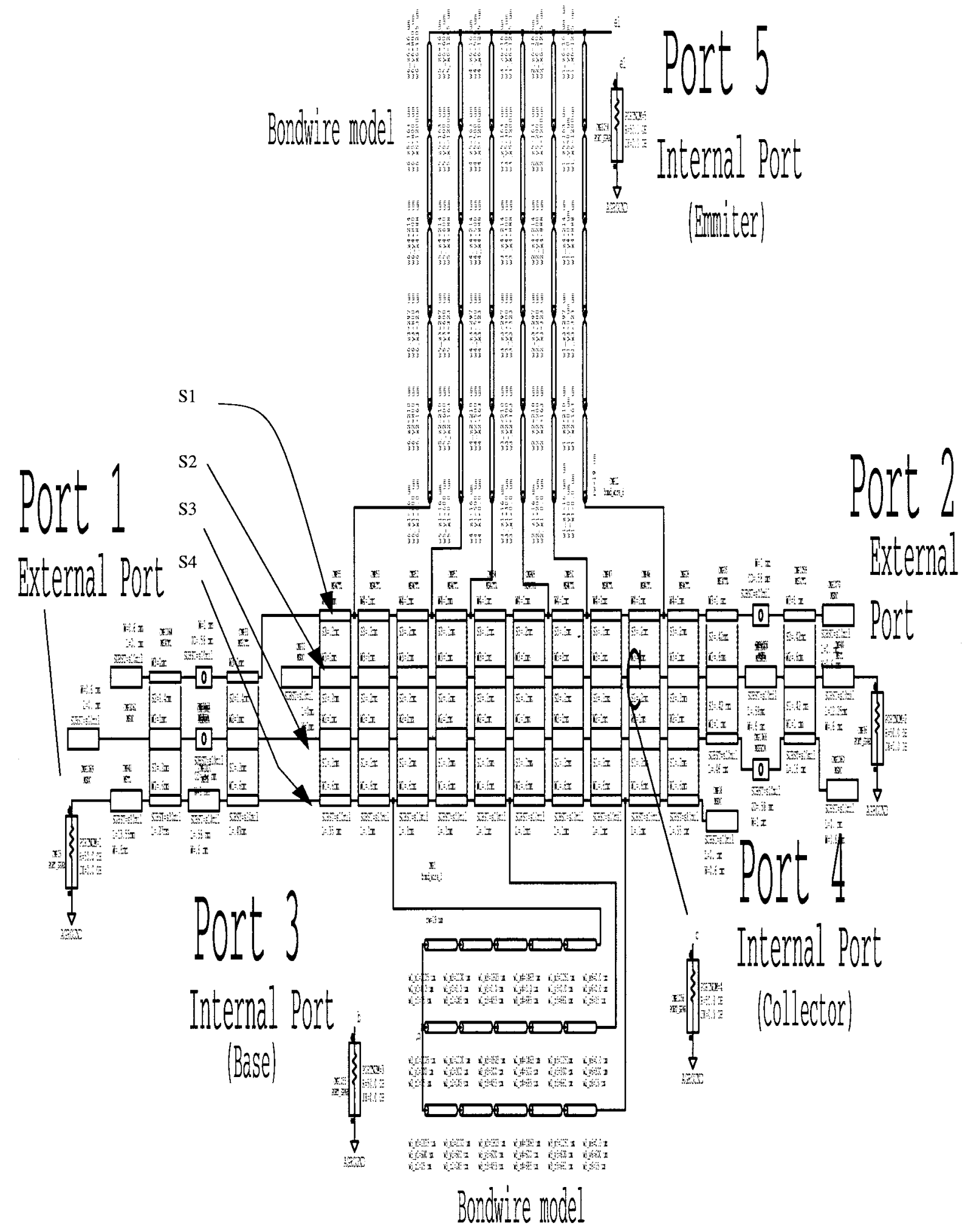

Fig. 4. The equivalent circuit for Fig. 1 implemented in MDS, consisting of coupled lines, Vias, and a model for the bondwire complex.

\section{GENERATing Dataset}

Generation of the five-port dataset is essential to the deembedding procedure. In order to construct a suitable model representative of the medium surrounding the floating two port (e.g., the transistor), one can use a combination of electromagnetic and circuit simulation programs including but not restricted to

- momentum, to provide an accurate model of (coupled) transmission lines;

- HFSS, to provide an accurate model of capacitance and connectors;

- MDS, to provide models of (coupled) transmission lines, inductors, etc.; 
TABLE I

The Verification Between the Original and the Deembedded Data. The Algorithms Implemented in MDS Are Given in the Appendix

\begin{tabular}{l||l||l||l||l}
\hline \hline \multirow{2}{*}{ Freq } & $\begin{array}{l}\text { Z11_Original - } \\
\text { Z11_Deembedded }\end{array}$ & $\begin{array}{l}\text { Z12_Original - } \\
\text { Z12_Deembedded }\end{array}$ & $\begin{array}{l}\text { Z21_Original - } \\
\text { Z21_Deembedded }\end{array}$ & $\begin{array}{l}\text { Z22_Original - } \\
\text { Z22_Deembedded }\end{array}$ \\
\hline \hline $0.8 \mathrm{GHz}$ & $0.48 \mathrm{e}-10-\mathrm{j} 0.25 \mathrm{e}-10$ & $-0.68 \mathrm{e}-15+\mathrm{j} 0.15 \mathrm{e}-15$ & $-0.68 \mathrm{c}-15+\mathrm{j} 015 \mathrm{c}-15$ & $0.75 \mathrm{c}-18-\mathrm{j} 0.29 \mathrm{c}-14$ \\
\hline \hline $1.2 \mathrm{GHz}$ & $0.92 \mathrm{e}-13-\mathrm{j} 0.91 \mathrm{e}-14$ & $-0.84 \mathrm{e}-13+\mathrm{j} 0.11 \mathrm{c}-14$ & $-0.78 \mathrm{c}-13+\mathrm{j} 0.84 \mathrm{c}-13$ & $-0.99 \mathrm{c}-17+0.31 \mathrm{e}-18$ \\
\hline \hline $1.6 \mathrm{GHz}$ & $-0.68 \mathrm{e}-13-\mathrm{j} 0.13 \mathrm{e}-14$ & $-0.77 \mathrm{e}-18+\mathrm{j} 0.25 \mathrm{c}-13$ & $-0.54 \mathrm{e}-18+0.91 \mathrm{c}-13$ & $0.91 \mathrm{c}-18-\mathrm{j} 0.15 \mathrm{e}-13$ \\
\hline \hline $2.0 \mathrm{GHz}$ & $0.29 \mathrm{e}-13+\mathrm{j} 0.11 \mathrm{e}-14$ & $0.16 \mathrm{e}-13+\mathrm{j} 0.17 \mathrm{e}-13$ & $0.12 \mathrm{e}-13-\mathrm{j} 0.15 \mathrm{c}-13$ & $-0.14 \mathrm{c}-17-\mathrm{j} 0.17 \mathrm{e}-17$ \\
\hline \hline $2.4 \mathrm{GHz}$ & $0.50 \mathrm{e}-18-\mathrm{j} 0.68 \mathrm{e}-18$ & $-0.33 \mathrm{e}-18+\mathrm{j} 0.31 \mathrm{e}-17$ & $-0.91 \mathrm{e}-18-\mathrm{j} 0.28 \mathrm{e}-13$ & $-0.11 \mathrm{e}-13+\mathrm{j} 0.16 \mathrm{e}-18$ \\
\hline \hline $2.8 \mathrm{GHz}$ & $0.17 \mathrm{e}-18-\mathrm{j} 0.67 \mathrm{e}-17$ & $-0.22 \mathrm{e}-18-\mathrm{j} 0.31 \mathrm{e}-17$ & $-0.27 \mathrm{e}-13-\mathrm{j} 0.31 \mathrm{e}-13$ & $0.17 \mathrm{e}-13+\mathrm{j} 0.61 \mathrm{e}-18$ \\
\hline \hline
\end{tabular}

- the Delft Coupled Bondwire Model (see [3]-[5]), to create a data set representing the five port.

\section{VERIFICATION} dure.

Using MDS we constructed a three step verification proce-

- First, we implemented a complete equivalent circuit for the medium surrounding the bipolar power transistor of Fig. 1. This equivalent circuit which is composed of coupled transmission lines, vias and bondwires (an internal Delft model) is depicted in Fig. 4.

- The related five-port dataset is generated using HP's MDS for a frequency range from 0.8 to $2.8 \mathrm{GHz}$.

- A two-port data set is connected to the internal ports 3-5, of the equivalent circuit, as illustrated in Fig. 4. Using MDS, the overall two-port impedance matrix is then determined.

- The deembedding algorithm describe above, as implemented in MDS, in combination with the five-port dataset generated in the previous step, is then used to deembed the data.

The embedding and deembedding procedure are uncorrelated so that the deembedded data provides a suitable control of the validity of the deembedding procedure. Table I illustrates the discrepancies found in comparing the original and the deembedded data.

\section{AN APPLICATION}

The extraction procedure has been carried out for an experimental Philips discrete bipolar power transistor, which is mounted on a substrate and connected by bondwires for characterization (see Fig. 1). In order to execute the new deembedding procedure, we used MDS to generate a dataset for the five-port network as described in the preceding section. We then import the dataset into HP's-parameter extraction software, IC-CAP, and execute in PEL (the internal IC-CAP language) implemented deembedding algorithms. Modeling of the mounting environment of the transistor is a two step process:
- CV measurements, are used to determine the bondpad capacitances, and

- the bondpad capacitances are then added to the equivalent circuit of the test structure.

\section{A. CV Measurement}

Depletion capacitance values are needed to properly extract the transistor parameters. The layout design is such that standard cv equipment cannot be used due to insurmountable calibration problems. In order to determine the capacitances (see Fig. 5) one can measure the scattering matrix under the special condition that $\mathrm{V}_{B E}<0.2 \mathrm{~V}, \mathrm{~V}_{C E}=0 \mathrm{~V}$. Next, convert the S-matrix to the impedance matrix, carry out deembedding and transform to the admittance matrix facilitating the calculation of the capacitances as follows:

$$
\begin{aligned}
C_{b e} & =\frac{\operatorname{Imag}\left(Y_{11}\right)+\operatorname{Imag}\left(Y_{21}\right)}{2 \pi f} \\
C_{b p e} & =\frac{\operatorname{Imag}\left(Y_{22}\right)+\operatorname{Imag}\left(Y_{21}\right)}{2 \pi f} \\
C_{b p b} & =\alpha C_{b p e} \\
C_{b c} & =\frac{-\operatorname{Imag}\left(Y_{21}\right)}{2 \pi f}-C_{b p b}
\end{aligned}
$$

where

$C_{b e}$ the base-emitter depletion capacitance;

$C_{b c}$ the base-collector depletion capacitance;

$C_{b p e}$ the emitter-collector bondpad capacitance;

$C_{b p b}$ the base-collector bondpad capacitance;

$\alpha \quad$ a process dependent constant.

\section{B. AC Measurements}

Adding the bondpad capacitances, we have:

- the emitter-collector bondpad capacitance, $C_{b p e}$, to ports 5 and 4 and

- the base-collector bondpad capacitance, $C_{b p b}$, to ports 4 and 3 , of the equivalent model shown in Fig. 4, one can generate a new data set for transistor parameter extraction. As an example the measured and deembedded " $f_{t}$ " curves 


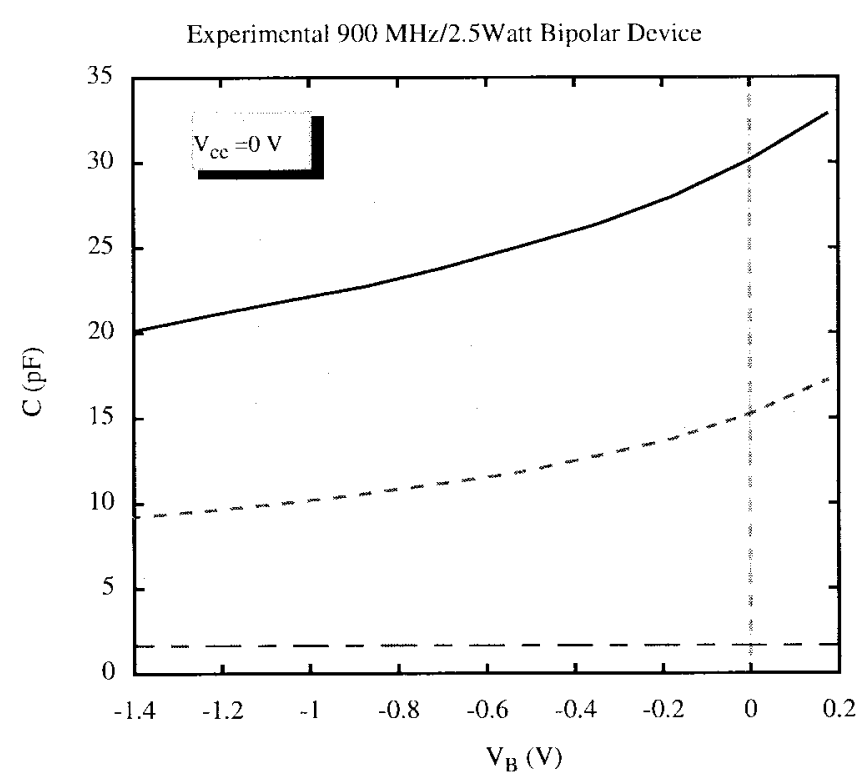

Base-emitter depletion capacitance

Base-collector depletion capacitance $\ldots . . .-$

Base-collector bondpad capacitance - - -

Fig. 5. Depletion capacitances and bondpad capacitances of the experimental bipolar power transistor.

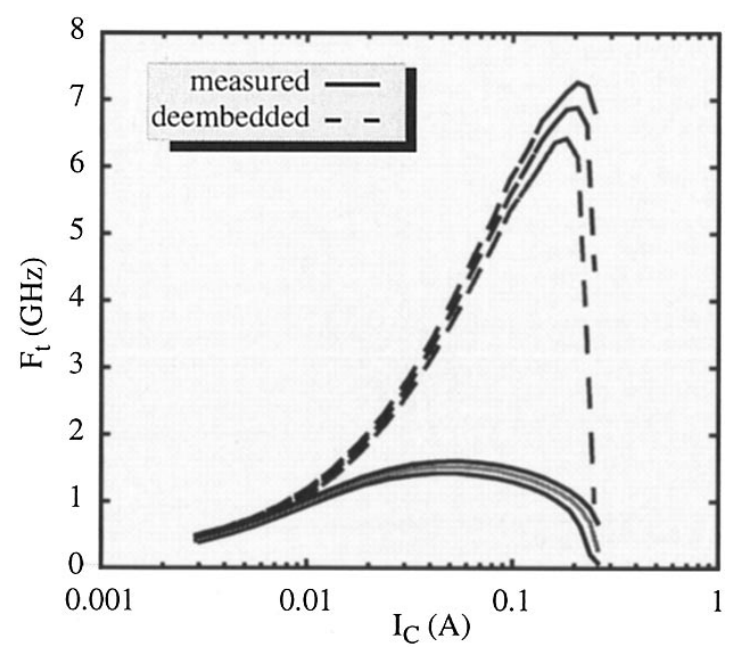

Fig. 6. Measured deembedded $f_{t}$ curves as a function of collector cureent.

are compared in Fig. 6. These deembedded parameters are then used in the modeling of the transistors.

\section{CONCLUSION}

A five-port generic algorithm that enables one to deembed high-frequency power transistor measurement data in one step, without compromise, regardless of the complex mounting environment of the transistor is introduced and illustrated with examples from practice. When using this method the accuracy of the deembedded data is primarily dependent on the exactness and accuracy of the models used to generate the five-port dataset.

\section{APPENDIX}

The DeEmbedding Algorithm ImPLemented IN MDS

$z m \_$The measured mounted transistor data.

$z f_{-}$- The simulated five-port dataset.

$z d r$ _ The deembedded data.

$$
\begin{aligned}
& \text { EQUATION } i d d 1 \\
& =(z m 21-z f 12)^{*} z m 12+(-z f 11+z m 11)^{*} z f 22 \\
& \quad+z m 22^{*} z f 11-z f 12^{*} z m 21+z f 12^{2}-z m 22^{*} z m 11 \\
& \text { EQUATION } z d r 11_{1} \\
& =\left((z f 13-z f 15)^{*} z f 23+(z m 21-z f 12)^{*} z f 55\right. \\
& \quad+(z m 21-z f 12)^{*} z f 33+(-z f 13+z f 15)^{*} z f 25 \\
& \left.\quad+\left(2^{*} z f 12-2^{*} z m 21\right)^{*} z f 35\right)^{*} z m 12
\end{aligned}
$$

EQUATION $z d r 11_{2}$

$$
\begin{aligned}
= & \left((-z f 11+z m 11)^{*} z f 55+(-z f 11+z m 11)^{*} z f 33\right. \\
& +\left(2^{*} z f 11-2^{*} z m 11\right)^{*} z f 35 \\
& \left.+z f 13^{2}+z f 15^{2}-2^{*} z f 13^{*} z f 15\right)^{*} z f 22 \\
& +(z f 11-z m 11)^{*} z f 23^{2}
\end{aligned}
$$

EQUATION $z d r 11_{3}$

$$
\begin{aligned}
= & \left(\left(-2^{*} z f 11+2^{*} z m 11\right)^{*} z f 25\right. \\
& +\left(-2^{*} z f 12+z m 21\right)^{*} z f 13 \\
& \left.+\left(-z m 21+2^{*} z f 12\right)^{*} z f 15\right)^{*} z f 23 \\
& +\left(z m 22^{*} z f 11-z f 12^{*} z m 21\right. \\
& \left.+z f 12^{2}-z m 22^{*} z m 11\right)^{*} z f 55
\end{aligned}
$$

EQUATION $z d r 11_{4}$

$$
\begin{aligned}
= & \left(z m 22^{*} z f 11-z f 12^{*} z m 21+z f 12^{2}\right. \\
& \left.-z m 22^{*} z m 11\right)^{*} z f 33+(z f 11-z m 11)^{*} z f 25^{2}
\end{aligned}
$$

EQUATION $z d r 11_{5}$

$$
\begin{aligned}
= & \left(\left(-z m 21+2^{*} z f 12\right)^{*} z f 13\right. \\
& \left.+\left(-2^{*} z f 12+z m 21\right)^{*} z f 15\right)^{*} z f 25 \\
& +\left(-2^{*} z m 22^{*} z f 11-2^{*} z f 12^{2}\right. \\
& \left.+2^{*} z m 22^{*} z m 11+2^{*} z f 12^{*} z m 21\right)^{*} z f 35 \\
& +2^{*} z m 22^{*} z f 13^{*} z f 15 \\
& -z m 22^{*} z f 15^{2}-z f 13^{2} * z m 22
\end{aligned}
$$

\section{EQUATION $z d r 11$}

$$
\begin{aligned}
= & \left(z d r 11_{1}+z d r 11_{2}+z d r 11_{3}\right. \\
& \left.+z d r 11_{4}+z d r 11_{5}\right) / i d d 1
\end{aligned}
$$

EQUATION $z d r 12_{1}$

$$
\begin{aligned}
= & \left((-z f 13+z f 15)^{*} z f 24+(-z m 21+z f 12)^{*} z f 55\right) \\
& +(z f 13-z f 15)^{*} z f 25+(z m 21-z f 12)^{*} z f 35 \\
& +(z f 34-z f 45)^{*} z f 12+z f 45^{*} z m 21 \\
& \left.-z m 21^{*} z f 34\right)^{*} z m 12
\end{aligned}
$$

EQUATION $z d r 12_{2}$

$$
\begin{aligned}
= & \left((z f 11-z m 11)^{*} z f 55+(-z f 11+z m 11)^{*} z f 35\right. \\
& +(-z f 14+z f 15)^{*} z f 13-z f 15^{2} \\
& +z f 14^{*} z f 15+(z f 34-z f 45)^{*} z f 11 \\
& \left.+z m 11^{*} z f 45-z m 11^{*} z f 34\right)^{*} z f 22
\end{aligned}
$$


EQUATION $z d r 12_{3}$

$$
\begin{aligned}
= & \left((-z f 11+z m 11)^{*} z f 24+(z f 11-z m 11)^{*} z f 25\right. \\
& +(z m 21-z f 12)^{*} z f 15-z f 14^{*} z m 21 \\
& \left.+z f 14^{*} z f 12\right)^{*} z f 23
\end{aligned}
$$

EQUATION $z d r 12_{4}$

$$
\begin{aligned}
= & \left((z f 11-z m 11)^{*} z f 25+z f 13^{*} z f 12\right. \\
& \left.-z f 12^{*} z f 15\right)^{*} z f 24+\left(-z f 12^{2}+z f 12^{*} z m 21\right. \\
& \left.-z m 22^{*} z f 11+z m 22^{*} z m 11\right)^{*} z f 55
\end{aligned}
$$

EQUATION $z d r 12_{5}$

$$
\begin{aligned}
= & (-z f 11+z m 11)^{*} z f 25^{2}+\left(-z f 13^{*} z f 12\right. \\
& +\left(-z m 21+2^{*} z f 12\right)^{*} z f 15 \\
& \left.-z f 14^{*} z f 12+z f 14^{*} z m 21\right)^{*} z f 25 \\
& +\left(z m 22^{*} z f 11-z f 12^{*} z m 21\right. \\
& \left.+z f 12^{2}-z m 22^{*} z m 11\right)^{*} z f 35
\end{aligned}
$$

EQUATION $z d r 126$

$$
\begin{aligned}
= & \left(-z m 22^{*} z f 15+z f 14^{*} z m 22\right)^{*} z f 13 \\
& +z m 22^{*} z f 15^{2}-z f 14^{*} z m 22^{*} z f 15 \\
& +\left(-z m 22^{*} z f 34+z m 22^{*} z f 45\right)^{*} z f 11 \\
& +(-z f 34+z f 45)^{*} z f 12^{2}
\end{aligned}
$$

EQUATION $z d r 12_{7}$

$$
\begin{aligned}
= & \left(z m 21^{*} z f 34-z f 45^{*} z m 21\right)^{*} z f 12 \\
& -z m 22^{*} z m 11^{*} z f 45+z m 22^{*} z m 11^{*} z f 34
\end{aligned}
$$

EQUATION $z d r 12$

$$
=\left(z d r 12_{1}+z d r 12_{2}+z d r 12_{3}+z d r 12_{4}+z d r 12_{5}\right.
$$$$
\left.+z d r 12_{6}+z d r 12_{7}\right) / i d d 1
$$

EQUATION $z d r 21_{1}$

$$
\begin{aligned}
= & \left((-z f 14+z f 15)^{*} z f 23+(-z m 21+z f 12)^{*} z f 55\right. \\
& +(-z f 15+z f 14)^{*} z f 25+(z m 21-z f 12)^{*} z f 35 \\
& +(z f 34-z f 45)^{*} z f 12 \\
& \left.+z f 45^{*} z m 21-z m 21^{*} z f 34\right)^{*} z m 12
\end{aligned}
$$

EQUATION $z d r 21_{2}$

$$
\begin{aligned}
= & \left((z f 11-z m 11)^{*} z f 55^{*}+(-z f 11+z m 11)^{*} z f 35\right. \\
& +(-z f 14+z f 15)^{*} z f 13-z f 15^{2} \\
& +z f 14^{*} z f 15+(z f 34-z f 45)^{*} z f 11 \\
& \left.+z m 11^{*} z f 45-z m 11^{*} z f 34\right)^{*} z f 22
\end{aligned}
$$

EQUATION $z d r 21_{3}$

$$
\begin{aligned}
= & \left((-z f 11+z m 11)^{*} z f 24+(z f 11-z m 11)^{*} z f 25\right. \\
& \left.+z f 14^{*} z f 12-z f 12^{*} z f 15\right)^{*} z f 23
\end{aligned}
$$

EQUATION $z d r 21_{4}$

$$
\begin{aligned}
= & \left((z f 11-z m 11)^{*} z f 25+(-z m 21+z f 12)^{*} z f 13\right. \\
& \left.+(z m 21-z f 12)^{*} z f 15\right)^{*} z f 24 \\
& +\left(-z f 12^{2}+z f 12^{*} z m 21-z m 22^{*} z f 11\right. \\
& \left.+z m 22^{*} z m 11\right)^{*} z f 55+(-z f 11+z m 11)^{*} z f 25^{2}
\end{aligned}
$$

EQUATION $z d r 21_{5}$

$$
\begin{aligned}
= & \left((z m 21-z f 12)^{*} z f 13+\left(-z m 21+2^{*} z f 12\right)^{*} z f 15\right. \\
& \left.-z f 14^{*} z f 12\right)^{*} z f 25+\left(z m 22^{*} z f 11\right. \\
& \left.-z f 12^{*} z m 21+z f 12^{2}-z n 22^{*} z m 11\right)^{*} z f 35
\end{aligned}
$$

EQUATION $z d r 21_{6}$

$$
\begin{aligned}
= & \left(-z m 22^{*} z f 15+z f 14^{*} z m 22\right)^{*} z f 13 \\
& +z m 22^{*} z f 15^{2}-z f 14^{*} z m 22^{*} z f 15 \\
& +\left(-z m 22^{*} z f 34+z m 22^{*} z f 45\right)^{*} z f 11 \\
& +(-z f 34+z f 45)^{*} z f 12^{2} \\
& +\left(z m 21^{*} z f 34-z f 45^{*} z m 21\right)^{*} z f 12 \\
& -z m 22^{*} z m 11^{*} z f 45+z m 22^{*} z m 11^{*} z f 34
\end{aligned}
$$

EQUATION $z d r 21$

$$
\begin{aligned}
= & z d r 21_{1}+z d r 21_{2}+z d r 21_{3} \\
& \left.+z d r 21_{4}+z d r 21_{5}+z d r 21_{6}\right) / i d d 1
\end{aligned}
$$

EQUATION $z d r 22_{1}$

$$
\begin{aligned}
= & \left(\left(z f 55+z f 44-2^{*} z f 45\right)^{*} z f 22-z f 25^{2}\right. \\
& +2^{*} z f 25^{*} z f 24-z m 22+z f 44 \\
& \left.-z m 22^{*} z f 55+2^{*} z m 22^{*} z f 45-z f 24^{2}\right)^{*} z m 11
\end{aligned}
$$

EQUATION $z d r 22_{2}$

$$
\begin{aligned}
= & \left(-2^{*} z f 14^{*} z f 15+z f 15^{2}+z f 14^{2}\right. \\
& \left.+2^{*} z f 11^{*} z f 45-z f 11^{*} z f 55-z f 11^{*} z f 44\right)^{*} z f 22 \\
& +z f 24^{2 *} z f 11+\left(-2^{*} z f 25^{*} z f 11\right. \\
& +\left(-z m 12-z m 21+2^{*} z f 12\right)^{*} z f 15 \\
& \left.-2^{*} z f 14^{*} z f 12+z f 14^{*} z m 21+z f 14^{*} z m 12\right)^{*} z f 24
\end{aligned}
$$

EQUATION $z d r 22_{3}$

$$
\begin{aligned}
= & \left(z m 22^{*} z f 11+z f 12^{2}+(-z m 21-z m 12)^{*} z f 12\right. \\
& \left.+z m 12^{*} z m 21\right)^{*} z f 44+\left(z m 22^{*} z f 11+z f 12^{2}\right. \\
& \left.+(-z m 21-z m 12)^{*} z f 12+z m 12^{*} z m 21\right)^{*} z f 55 \\
& +z f 25^{2 *} z f 11
\end{aligned}
$$

EQUATION $z d r 22_{4}$

$$
\begin{aligned}
= & \left(\left(z m 21-2^{*} z f 12+z m 12\right)^{*} z f 15-z f 14^{*} z m 12\right. \\
& \left.-z f 14^{*} z m 21+2^{*} z f 14^{*} z f 12\right)^{*} z f 25 \\
& -z m 22^{*} z f 15^{2}+2^{*} z f 14^{*} z m 22^{*} z f 15 \\
& -2^{*} z m 22^{*} z f 11^{*} z f 45-2^{*} z f 12^{2 *} z f 45
\end{aligned}
$$

EQUATION $z d r 22_{\check{5}}$

$$
\begin{aligned}
= & \left(2^{*} z m 12^{*} z f 45+2^{*} z f 45^{*} z m 21\right)^{*} z f 12 \\
& -2^{*} z m 12^{*} z f 45^{*} z m 21-z f 14^{2 *} z m 22
\end{aligned}
$$

EQUATION $z d r 22$

$$
\begin{aligned}
= & \left(z d r 22_{1}+z d r 22_{2}+z d r 22_{3}\right. \\
& \left.+z d r 22_{4}+z d r 22_{5}\right) / i d d 1
\end{aligned}
$$

\section{ACKNOWLEDGMENT}

The authors would like to thank the help of many people who made this work possible. In particular, H. J. ten Dolle, Philips Semiconductors, discussed the application and provided the bipolar power transistors and the required test structures and K. Mouthaan, Delft University of Technology, helped with the bondwire calculations (see Fig. 4 and [3]).

\section{REFERENCES}

[1] A. Cho and D. E. Burk, "A three-step method for the deembedding of high frequency S-parameters measurement," IEEE Trans. Electron Devices, vo1. 38, pp. 1371-1375, June 1991. 
[2] J. Weng, "A universal deembedding procedure for the 'on-wafer' $\mathrm{GHz}$ probing," IEEE Trans. Electron Devices, vo1. 42, pp. 1703-1705, Sept. 1995.

[3] K. Mouthaan, R. Tinti, M. de Kok, H. C. de Graaf, J. L. Tauritz, and J. Slotboom, "Microwave modeling and measurement of the self- and mutual inductance of coupled bondwires," in Proceedings of the 1997 Bipolar/BiCMOS Circuit and Technology Meeting, Minneapolis, MN, USA, Sept. 1997, pp. 166-169.

[4] A. O. Harm, K. Mouthaan, E. Aziz, and M. Versleijen, "Modeling and simulation of hybrid RF circuits using a versatile compact bond wire model," in Proc. 28th European Microwave Conf., Amsterdam, The Netherlands, Oct. 1998, vo1. 2, pp. 529-535.

[5] K. Mouthaan, R. Tinti, H. C. de Graaf, J. L. Tauritz, and J. Slotboom, "Microwave modeling and measurement of prematch circuitry for RF power transistors," in Proc. MTT-S European Wireless, Amsterdam, The Netherlands, Oct. 1998, pp. 198-204.

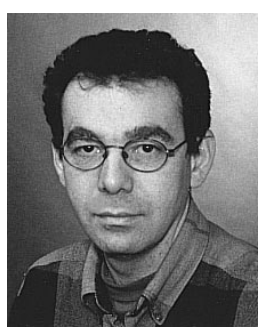

Reza Mahmoudi was born in Brojerd, Iran, on June 2, 1965. He received the M.Sc. degree with a thesis entitled, "A Measurement System for Transistor Noise Parameters" and the Designer's Certificate with a thesis entitled, "A Systematic Design Method for a Feedforward Error Control System" from the Delft University of Technology, The Netherlands, in 1993 and 1996, respectively. He is currently working towards the Ph.D. degree in the area of "multidisciplinary design method" with the Microwave Component Group of the Delft Institute of Microelectronics and Submicron Technology, The Netherlands.

His interests are in high-frequency noise, microwave deembedding techniques, transistor modeling, communication systems simulation, realization, and testing.

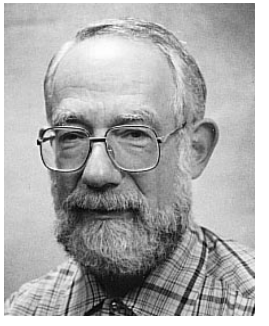

Joseph L. Tauritz (S'60-M'63) was born in Brooklyn, NY, in 1942. He received the B.E.E. degree from New York University, New York, NY, in 1963 and the M.S.E. degree in electrical engineering from the University of Michigan, Ann Arbor, in 1968.

From 1970 to 1971, he was a Research Fellow at the Delft University of Technology, The Netherlands. In 1971, he joined the scientific staff of the Faculty of Electrical Engineering, where he is presently an Associate Professor. Since 1976, he has headed the Microwave Component Group, where he is principally concerned with the systematic application of computer-aided design techniques in research and education. He first became acquainted with microwaves while working as a Junior Engineer on circularly polarized antennas at Wheeler Laboratories in the summer of 1962. From 1963 to 1970, he worked as a Technical Specialist attached to the RF Department of the Conduction Corporation. His interests include the modeling of high-frequency components for use in the design of MIC's and MMIC's, filter synthesis, and planar superconducting microwave components.

Mr. Tauritz is a member of Eta Kappa Nu. 